\title{
Acupuncture as an adjunctive therapy for arrhythmia: a Delphi expert consensus survey
}

\author{
Jin-Ling Li", Li-Qiong Wang", Na Zhang, Xin-Tong Su, Ying Lin, Jing-Wen Yang, Guang-Xia Shi, \\ Cun-Zhi Liu
}

International Acupuncture and Moxibustion Innovation Institute, School of Acupuncture-Moxibustion and Tuina, Beijing University of Chinese Medicine, Beijing, China

Contributions: (I) Conception and designed: CZ Liu, LQ Wang, JL Li; (II) Administrative support: CZ Liu; (III) Provision of study materials or patients: JW Yang, GX Shi; (IV) Collection and assembly of data: Y Lin, XT Su; (V) Data analysis and interpretation: JL Li, N Zhang, LQ Wang; (VI) Manuscript writing: All authors; (VII) Final approval of manuscript: All authors.

\#These authors contributed equally to this work.

Correspondence to: Prof. Cun-Zhi Liu. International Acupuncture and Moxibustion Innovation Institute, School of Acupuncture-Moxibustion and Tuina, Beijing University of Chinese Medicine, No. 11, Bei San Huan Dong Lu, Chaoyang District, Beijing 100029, China. Email: 1cz623780@126.com.

\begin{abstract}
Background: Current evidence suggests that acupuncture is an effective adjunctive therapy that can bring potential benefits to patients with cardiac arrhythmias. However, there are relevant gaps in the optimal therapeutic strategy, which may cause uncertainties on the best practice of acupuncture treatment for arrhythmia. We aim to develop consensus-based recommendations for clinical guidance on acupuncture treatment of cardiac arrhythmias.

Methods: A multidisciplinary panel of specialists was invited to participate in a two-round semi-open clinical issue investigation. Meanwhile, relevant literature reviews were searched in 3 databases to provide evidence. Subsequently, an initial consensus voting list on acupuncture as an adjunctive therapy for cardiac arrhythmias was derived from the clinical investigation and literature review. Finally, 30 authoritative experts reached a consensus on the key issues of the voting list by a three-round modified Delphi survey. Consensus was defined when $>80 \%$ agreement was achieved.

Results: Following the three-round Delphi survey, there were 32 items (91.43\%) finally reaching consensus, including the following 5 domains: (I) the benefits of acupuncture for the appropriate population; (II) the general therapeutic principle; (III) the acupuncture strategy; (IV) the relevant adverse events; (V) others.

Conclusions: Consensus was achieved on some key elements. Given the lack of guidelines and the substantial heterogeneity of previous studies, these recommendations are of value in providing guidance for clinical practice of acupuncturists and in assisting patients with arrhythmia to obtain standardized acupuncture treatment. It also pointed out some problems that need to be carefully explored in future studies.
\end{abstract}

Keywords: Acupuncture; cardiac arrhythmia; expert consensus; adjunctive therapy; Delphi study

Submitted Mar 31, 2021. Accepted for publication Jul 28, 2021.

doi: $10.21037 / \mathrm{cdt}-21-201$

View this article at: https://dx.doi.org/10.21037/cdt-21-201

\section{Introduction}

Cardiac arrhythmia (CA) is a family of cardiac conditions characterized by irregular rate or rhythm of heartbeats (1). There are many types of $\mathrm{CA}$ with various manifestations and hazards, such as sinus/atrial/ventricular arrhythmia, or tachyarrhythmia/bradyarrhythmia. The overall prevalence of CA is about $3.4 \%$ (2), and approximate 3.7 million people die of CA annually worldwide (3). There are many important factors that increase the risk of CA, such as 


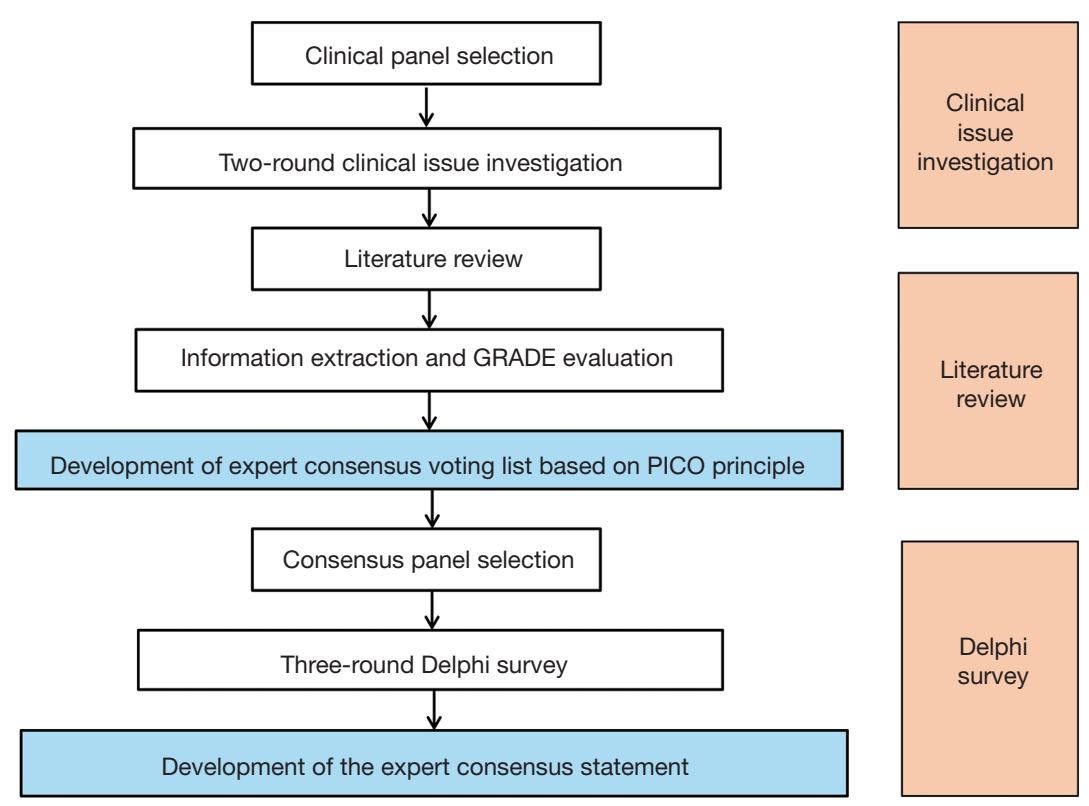

Figure 1 The development process of expert consensus. GRADE, Grading of Recommendations, Assessment, Development, and Evaluation; PICO, population, intervention, control, and outcomes.

aging, heredity, alcohol and tobacco use, physical inactivity, and obesity (4-10). In the context of an aging society, the increasing prevalence of $\mathrm{CA}$ is bringing a growing challenge to the global health-care system. It is urgent and of great significance to manage CA effectively, since CA is regarded as a leading cause of morbidity and mortality (11).

Conventional management depends on CA classifications, which mainly includes antiarrhythmic drugs, surgical treatment, catheter ablation, electrical cardioversion, and device implantation (12-14). However, to some extent, these therapies are plagued by insufficient effectiveness $(15,16)$, limited indications, as well as conspicuous adverse effects and complications (17-19). The conventional management of CA brings health benefits to patients, but may be associated with a substantial financial burden (20). The limitations associated with conventional arrhythmia management have aroused interest in seeking an effective, inexpensive, and safe complementary intervention.

Acupuncture, as an ancient therapeutic method of traditional Chinese medicine (TCM), has a long-term practical history in treating palpitations (one of the main symptoms of arrhythmia). Currently, evidence has shown that acupuncture as an adjunctive therapy could produce benefits for patients with CA, especially in regulating the rate and rhythm of heartbeats (21-27). As a complex intervention model, certain parameters of acupuncture (selection of acupoints, frequency of acupuncture, and duration of needle retention, etc.) are the important factors associated with the therapeutic effects (28). However, the acupuncture parameters applied in different clinical studies are distinct from each other, which may be a key reason for the inconsistent clinical effects. It is imperative to determine the optimal acupuncture parameters for CA treatment.

The uncertainties regarding acupuncture's therapeutic recommendations for CA has motivated extensive investigations on clinical issues of concern and prompted us to establish an expert consensus on this topic using a Delphi survey. The primary purpose was to optimize the acupuncture therapeutic strategy for better effectiveness and provide practical recommendations for future clinical practice and trials.

\section{Methods}

The development of the expert consensus consisted of the four major stages: (I) setting up an assistant team; (II) clinical issue investigation; (III) literature review; (IV) Delphi survey. The development of the expert consensus is shown in Figure 1. 


\section{Setting up an assistant team}

An assistance team was responsible for the design, analysis, and interpretation of the questionnaires and voting lists. The assistance team consisted of one senior acupuncture expert (CZ Liu), two clinical acupuncturists (N Zhang, and JL Li), two methodologists (Wei Chen and LQ Wang) and two research secretaries (XT Su and Y Lin).

\section{Clinical issue investigation}

\section{Development of the clinical questionnaire}

After reviewing previous literature on acupuncture for CA, the assistance team came up with an initial clinical questionnaire by having several video conferences and group discussions. Subsequently, three acupuncturists (Hui $\mathrm{Hu}$, Lin Du, and Y Lin) and two methodologists (Wei Chen and LQ Wang) were invited to revise the initial clinical questionnaire. According to their suggestions, the initial clinical questionnaire was modified by the assistance team. To gather a broader perspective on therapeutic suggestions, the modified clinical questionnaire was designed in a semiopen format.

\section{Selection of the clinical panel}

Given that acupuncture is related to empirical medicine, it is a critical step to collect pragmatic recommendations on clinical issues of concern to clinicians. Therefore, a group of acupuncturists from the China Association of AcupunctureMoxibustion and cardiologists with clinical experience in the management of arrhythmia were convened to participate in the investigation of clinical issues. In addition, to further enrich the relevant questionnaires, they were also required to share relevant suggestions to facilitate the optimization of the later questionnaires.

\section{Process of clinical issue investigation}

The two-round clinical issue investigation was conducted from February 4, 2020 to March 24, 2020. The clinical questionnaire was sent to the clinical panel via online software (www.wjx.cn, a professional online questionnaire survey, evaluation, and voting platform). After analyzing the results of the first round, the assistant team formulated the second-round questionnaire based on the feedback from round 1 and sent it to the same panel again. By summarizing the results and the feedback of the two-round investigation, an initial expert consensus voting list was drafted in accordance with the population, intervention, control, and outcomes (PICO) principle.

\section{Literature review}

To further supplement the expert consensus voting list, literature retrieval was conducted to provide evidence for the voting list. The systematic review on acupuncture treatment of CA was searched in PubMed, Embase and Cochrane Library databases from January 2000 to March 2020. Titles and abstracts were searched according to the following search terms: (acupuncture OR electroacupuncture OR electro-acupuncture) AND (arrhythmia OR arrhythmias OR cardiac arrhythmia OR cardiac arrhythmias OR Tachycardia OR bradycardia OR sick sinus syndrome OR premature beat OR sinus arrest OR conduction block OR atrial fibrillation OR atrial flutter OR ventricular flutter OR ventricular fibrillation OR Wolff-Parkinson-White syndrome) AND (review OR systematic review OR metaanalysis). Only systematic reviews restricted to English publications and full-text available were included. Two independent researchers extracted information and assessed the quality of clinical evidence under the guidance of the Grading of Recommendations, Assessment, Development, and Evaluation (GRADE). The quality of evidence was assessed as high certainty, moderate certainty, low certainty, very low certainty. The discrepancies between the two researchers were resolved by a third researcher. During the Delphi survey stage, the evidence from the reviews was appended to the corresponding items of the voting list for the panelists to make decisions.

\section{Delphi survey}

\section{Selection of the consensus panel}

Veteran acupuncture experts were convened to set up a consensus panel for a three-round Delphi survey. These experts had to be authoritative scholars with deputy senior titles or above, and have considerable experience in the acupuncture treatment of CA. The invitation letters were sent to these experts in advance via e-mail or WeChat (a universal Chinese instant messaging application) to ensure their willing in participating the survey. After confirmation of their attitudes, the Delphi surveys were conducted immediately.

\section{Process of the Delphi survey}

Delphi survey is an accepted scientific technique with a structured process $(29,30)$. The consensus was conducted 
on the basis of the modified Delphi method, using an approach similar to the frameworks for other consensus on non-pharmaceutical therapy (31-33). The expert consensus voting list, generated from the clinical issue investigation and the literature review, was sent to the consensus panel via online software (www.wjx.cn) from April 5 to 25, 2020. Panelists were instructed to record their responses as agreement, neutrality or disagreement for each item according to their experience and current evidence. Each item on the voting list was attached to a comment box to gather explanation and comment on the choice. The feedback from each round was fully utilized to optimize the items for the next round of Delphi survey. The consensus was defined when $>80 \%$ agreement on a given item was reached (34). Otherwise, it was considered to be a controversial item. Based on the previous round of Delphi survey, the controversial items ( $\leq 80 \%$ agreement) would proceed to the next round of consensus voting. The expert consensus statements were achieved by the three rounds of the Delphi survey.

\section{Formulation of the expert consensus statements}

The assistant team drafted a manuscript on the expert consensus statements based on the three-round Delphi survey and relevant suggestions shared by panelists. Subsequently, the manuscript was sent back to the consensus panel for reconfirmation. After discussions and revisions, the final expert consensus statements were formulated.

\section{Statistical analysis}

The composition characteristics of the two panels (such as gender, years of clinical experience, professional title, geographical distribution, etc.) will be described as the percentage. For the results of the three-round of Delphi Survey, the proportion of "agreement", "neutrality" and "disagreement" in each round was calculated respectively, and the consensus results were described as the percentage. The proportion was observed when more than $80 \%$, so that the consensus could be reached on the "agreement", "neutrality" and "disagreement". The data was analyzed by SPSS software.

\section{Results}

\section{Results of the two-round clinical issue investigation}

A total of 70 individuals from different administrative regions of China were initially invited to participate in the clinical issue investigation. Fifty-nine clinical specialists (response rate: $84 \%$ ) who expressed their willingness to participate the first round of investigation, and 53 of them completed the second round. More than half of these clinical specialists have more than 10 years of clinical experience. The detailed characteristics of the clinical panel are shown in Table 1.

After synthesizing the two-round clinical issue investigation, an initial expert consensus voting list with 35 items was generated and formulated in accordance with the PICO principle (as shown in Table 2). According to the feedbacks of the clinical specialists, the primary contents of the voting list included appropriate disease subtypes, acupuncture principles, acupuncture strategies, possible benefits, and adverse events.

\section{Results of literature review}

In the process of the literature review, only four systematic reviews were finally eligible according to our screening criteria $(12,26,27,35)$. The quality of evidence from these systematic reviews were rated "very low" by GRADE standard. The key information of these systematic reviews was extracted and subsequently attached as evidence to the corresponding items on the initial expert consensus voting list. Items without available evidence were accompanied by the results of the two-round clinical issue investigation.

\section{Results of the three-round Delphi survey}

The Delphi survey was conducted in three rounds. A total of 30 panelists (response rate: 100\%) completed the first round of Delphi survey, and 29 (96.67\%) panelists accomplished the second round, and $30(100 \%)$ panelists completed the third round of the survey. Nearly $90 \%$ of the panelists had senior titles and over $65 \%$ have at least 20 -year acupuncture practical experience. The detailed characteristics of the consensus panel are shown in Table 1. 
Table 1 Characteristics of the two panels

\begin{tabular}{|c|c|c|c|c|c|}
\hline \multirow{3}{*}{ Variable } & \multicolumn{5}{|c|}{ Expert } \\
\hline & \multicolumn{2}{|c|}{ Clinical panel } & \multicolumn{3}{|c|}{ Consensus panel } \\
\hline & Round 1 & Round 2 & Round 1 & Round 2 & Round 3 \\
\hline Women, n (\%) & $35(59.53)$ & $33(62.26)$ & $11(36.67)$ & $10(34.48)$ & $11(36.67)$ \\
\hline \multicolumn{6}{|c|}{ Geographical distribution, n (\%) } \\
\hline Eastern China & $18(30.51)$ & 18 (33.96) & $8(26.67)$ & $8(27.59)$ & $8(26.67)$ \\
\hline Southern China & $3(5.08)$ & $3(5.66)$ & $1(3.33)$ & $1(3.45)$ & $1(3.33)$ \\
\hline North China & $18(30.51)$ & $16(30.19)$ & $11(36.67)$ & $11(37.93)$ & $11(36.67)$ \\
\hline Central China & $3(5.08)$ & $3(5.66)$ & $4(13.33)$ & $4(13.79)$ & $4(13.33)$ \\
\hline Northeast China & $11(18.64)$ & $9(16.98)$ & $3(10.00)$ & $2(6.90)$ & $3(10.00)$ \\
\hline Southwest China & $4(6.78)$ & $2(3.77)$ & $3(10.00)$ & $3(10.34)$ & $3(10.00)$ \\
\hline Northwest China & $2(3.39)$ & $2(3.77)$ & $0(0.00)$ & $0(0.00)$ & $0(0.00)$ \\
\hline \multicolumn{6}{|c|}{ Highest qualification, n (\%) } \\
\hline Bachelor's degree & $3(5.08)$ & $3(5.66)$ & $2(6.67)$ & $2(6.90)$ & $2(6.67)$ \\
\hline Master's degree & $17(28.81)$ & $15(28.30)$ & $7(23.33)$ & $7(24.14)$ & $7(23.33)$ \\
\hline $\mathrm{PhD}$ & $39(66.10)$ & $35(66.04)$ & $21(70.00)$ & $20(68.97)$ & $21(70.00)$ \\
\hline \multicolumn{6}{|c|}{ Professional title, n (\%) } \\
\hline Intermediate title & $14(23.73)$ & $12(22.64)$ & $0(0.00)$ & $0(0.00)$ & $0(0.00)$ \\
\hline Deputy senior title & $25(42.37)$ & $21(39.62)$ & $3(10.00)$ & $3(10.34)$ & $3(10.00)$ \\
\hline Senior title & $20(33.90)$ & $20(37.74)$ & $27(90.00)$ & $26(89.66)$ & $27(90.00)$ \\
\hline \multicolumn{6}{|c|}{ Acupuncture practical experience (years), n (\%) } \\
\hline $3-10$ years & $18(30.51)$ & $15(28.30)$ & $1(3.33)$ & $1(3.45)$ & $1(3.33)$ \\
\hline $11-20$ years & $31(52.54)$ & 28 (52.83) & $9(30.00)$ & $9(31.03)$ & $9(30.00)$ \\
\hline $21-30$ years & $8(13.56)$ & $8(15.09)$ & $8(26.67)$ & $8(27.59)$ & $8(26.67)$ \\
\hline$>30$ years & 2 (3.39) & $2(3.77)$ & $12(40.00)$ & $11(37.93)$ & $12(40.00)$ \\
\hline
\end{tabular}

In the first round of voting, consensus ( $>80 \%$ agreement) was reached on 25 of 35 items presented on the voting list (Figure 2). Ten controversial items ( $\leq 80 \%$ agreement) were further assessed in round 2. And four items reached $>80 \%$ agreement in the second round (Figure 3). The remaining six items were evaluated in the third round, and three items were achieved the consensus (Figure 4). On the basis of the panelists' feedbacks, no additional item was proposed to the list during the three-round survey. Totally, 32 items were compiled into the final expert consensus statements, and three controversial items were excluded after the three-round Delphi survey.

\section{Formulation of the final expert consensus statements}

The final expert consensus included 32 items that covered the topics of concern for the acupuncture treatment of arrhythmia. These items were classified into five domains: (I) the possible benefits of acupuncture on appropriate disease subtypes; (II) the general principle, such as syndrome differentiation and treatment; (III) the therapeutic strategy, mainly containing acupoints selection, frequency and duration, etc.; (IV) the common adverse events (e.g., subcutaneous hematoma); (V) others, such as the qualifications of acupuncturists. 
Table 2 The expert consensus voting list

No. Key items

1 Acupuncture is recommended as an adjunctive therapy for arrhythmias caused by cardiac diseases (coronary atherosclerotic heart disease, hypertension, cardiomyopathies, etc.)

2 Acupuncture is recommended as an adjunct therapy for arrhythmia caused by non-cardiogenic internal diseases (respiratory system diseases, and digestive diseases)

3 Acupuncture is recommended as an adjunct therapy for arrhythmia caused by lifestyle-related factors (over exercise, excessive emotion, drinking coffee, smoking and drinking, etc.)

4 For patients with sinus arrhythmia, acupuncture is recommended as an adjunctive therapy to relieve clinical symptoms (palpitations, dyspnea or shortness of breath, chest pain or discomfort, fatigue, and dizziness)

5 For patients with sinus arrhythmia, acupuncture is recommended as an adjunctive therapy to regulate the rate or rhythm of heartbeat (such as reducing frequency or duration of arrhythmia attacks, and decreasing the time of restoration to NSR)

6 For patients with sinus arrhythmia, acupuncture is recommended as an adjunctive therapy to improve the quality of life and mental conditions

7 For patients with atrial arrhythmia, acupuncture is recommended as an adjunctive therapy to relieve clinical symptoms (palpitations, dyspnea or shortness of breath, chest pain or discomfort, fatigue, and dizziness)

8 For patients with atrial arrhythmia, acupuncture is recommended as an adjunctive therapy to regulate the rate or rhythm of heartbeat (such as reducing frequency or duration of arrhythmia attacks, and decreasing the time of restoration to NSR)

9 For patients with atrial arrhythmia, acupuncture is recommended as an adjunctive therapy to improve the quality of life and mental conditions

10 For patients with tachyarrhythmia, acupuncture is recommended to as an adjunctive therapy relieve clinical symptoms (palpitations, dyspnea or shortness of breath, chest pain or discomfort, fatigue, and dizziness)

11 For patients with tachyarrhythmia, acupuncture is recommended to as an adjunctive therapy regulate the rate or rhythm of heartbeat (such as reducing frequency or duration of arrhythmia attacks, and decreasing the time of restoration to NSR)

12 For patients with tachyarrhythmia, acupuncture is recommended as an adjunctive therapy to improve the quality of life and mental conditions

13 For patients with bradyarrhythmia, acupuncture is recommended as an adjunctive therapy to relieve clinical symptoms (palpitations, dyspnea or shortness of breath, chest pain or discomfort, fatigue, and dizziness)

14 For patients with bradyarrhythmia, acupuncture is recommended as an adjunctive therapy to regulate the rate or rhythm of heartbeat (such as reducing frequency or duration of arrhythmia attacks, and decreasing the time of restoration to NSR)

15 For patients with bradyarrhythmia, acupuncture is recommended as an adjunctive therapy to improve the quality of life and mental conditions

16 For patients with atrial fibrillation, acupuncture is recommended as an adjunctive therapy to relieve clinical symptoms (palpitations, dyspnea or shortness of breath, chest pain or discomfort, fatigue, and dizziness)

17 For patients with atrial fibrillation, acupuncture is recommended as an adjunctive therapy to regulate the rate or rhythm of heartbeat (such as reducing frequency or duration of arrhythmia attacks, and decreasing the time of restoration to NSR)

18 For patients with atrial fibrillation, acupuncture is recommended as an adjunctive therapy to improve the quality of life and mental conditions

19 For patients who underwent invasive treatment (such as catheter ablation, pacemaker implantation) in arrhythmia, acupuncture is recommended as an adjunctive therapy to reduce the recurrence of arrhythmia

20 For patients who underwent invasive treatment (such as catheter ablation, pacemaker implantation) in arrhythmia, acupuncture is recommended as an adjunctive therapy to reduce the incidence of related cardiovascular events

21 For patients who underwent invasive treatment (such as catheter ablation, pacemaker implantation) in arrhythmia, acupuncture is recommended as an adjunctive therapy to improve the quality of life and mental conditions

Table 2 (continued) 
Table 2 (continued)

\begin{tabular}{|c|c|}
\hline No. & Key items \\
\hline 22 & The relief of clinical symptoms can be maintained for 1-6 months after acupuncture treatment \\
\hline 23 & $\begin{array}{l}\text { The traditional Chinese medicine theory of syndrome differentiation and treatment is recommended to be carefully considered in the } \\
\text { treatment of arrhythmia, especially for heart-yang stasis deficiency syndrome, qi-yin deficiency syndrome, qi stagnation and blood } \\
\text { stasis syndrome, phlegm block syndrome etc. }\end{array}$ \\
\hline 24 & It is recommended to select acupoints on Shoujueyin pericardial meridian and Shoushaoyin pericardial meridian. \\
\hline 26 & The recommended types of specific acupoints are: Beishu acupoint and Yuan acupoint \\
\hline 27 & The recommended specific acupoints combinations are: Yuan-Luo combination, and Beishu-Mu combination \\
\hline 28 & The recommended acupuncture manipulation is the even reinforcing-reducing method \\
\hline 31 & The commended acupuncture frequency is 3 sessions per week \\
\hline 32 & The recommend acupuncture course is 4 weeks \\
\hline 33 & Deqi is an impartment factor for the achievement of therapeutic effect \\
\hline 34 & $\begin{array}{l}\text { Adverse events are uncommon in the acupuncture treatment of arrhythmia. Possible adverse events include subcutaneous } \\
\text { hematoma and abnormal post-acupuncture sensation (such as pain, numbness) }\end{array}$ \\
\hline 35 & It is recommended that acupuncture practitioners should hold the license of TCM and at least 3 years of experience in acupuncture \\
\hline
\end{tabular}

NSR, normal sinus rhythm; TCM, traditional Chinese medicine.

\section{Discussion}

This study aims to achieve an expert consensus on some specific issues of acupuncture treatment for arrhythmia by means of Delphi survey and resolved the most concerned issues of clinicians. In this consensus, experts agree that acupuncture can be a potential adjunctive therapy for arrhythmia. The results show high level of agreement amongst panelists for most items (32 of 35), especially in acupuncture parameters and influencing factors. A relatively optimized acupuncture strategy of arrhythmia has been formulated, which provides a certain reference for future clinical practice and trials. Considering the theoretical background of acupuncture, the consensus contains many items relates to the concept of TCM. Nevertheless, this consensus still has generalization and application.

According to our consensus, acupuncture was recommended as an adjunctive therapy to regulate the rate or rhythm of heartbeats for patients with arrhythmia. This is similar to the results of previous studies. $\mathrm{Li}$ et al. found that acupuncture plus oral antiarrhythmic drugs contributed to a significant benefit on the rate or rhythm of heartbeat for patients with ventricular premature beats compared to oral antiarrhythmic drugs alone (12). Another meta-analysis showed that acupuncture combined with intravenous deslanoside had an additive effect on the response rate than intravenous deslanoside alone (35).

It is worth noting that experts agreed that acupuncture can improve the quality of life and clinical symptoms for all types of arrhythmias expect atrial fibrillation in the first round, while it was not until the third round that consensus was agreed on improvement of rate and rhythm. This suggests that experts initially had doubts whether acupuncture could improve the objective arrhythmia parameters. Due to the low quality of current evidence, a robust conclusion cannot be drawn. Therefore, experts were more cautious when interpreted the results. In addition, acupuncturists know that acupuncture could improve the clinical symptoms such as palpitations and chest discomfort by communication with patients. However, due to the lack of relatively professional examinations (electrocardiogram, etc.) in the acupuncture clinics, experts are uncertain about 


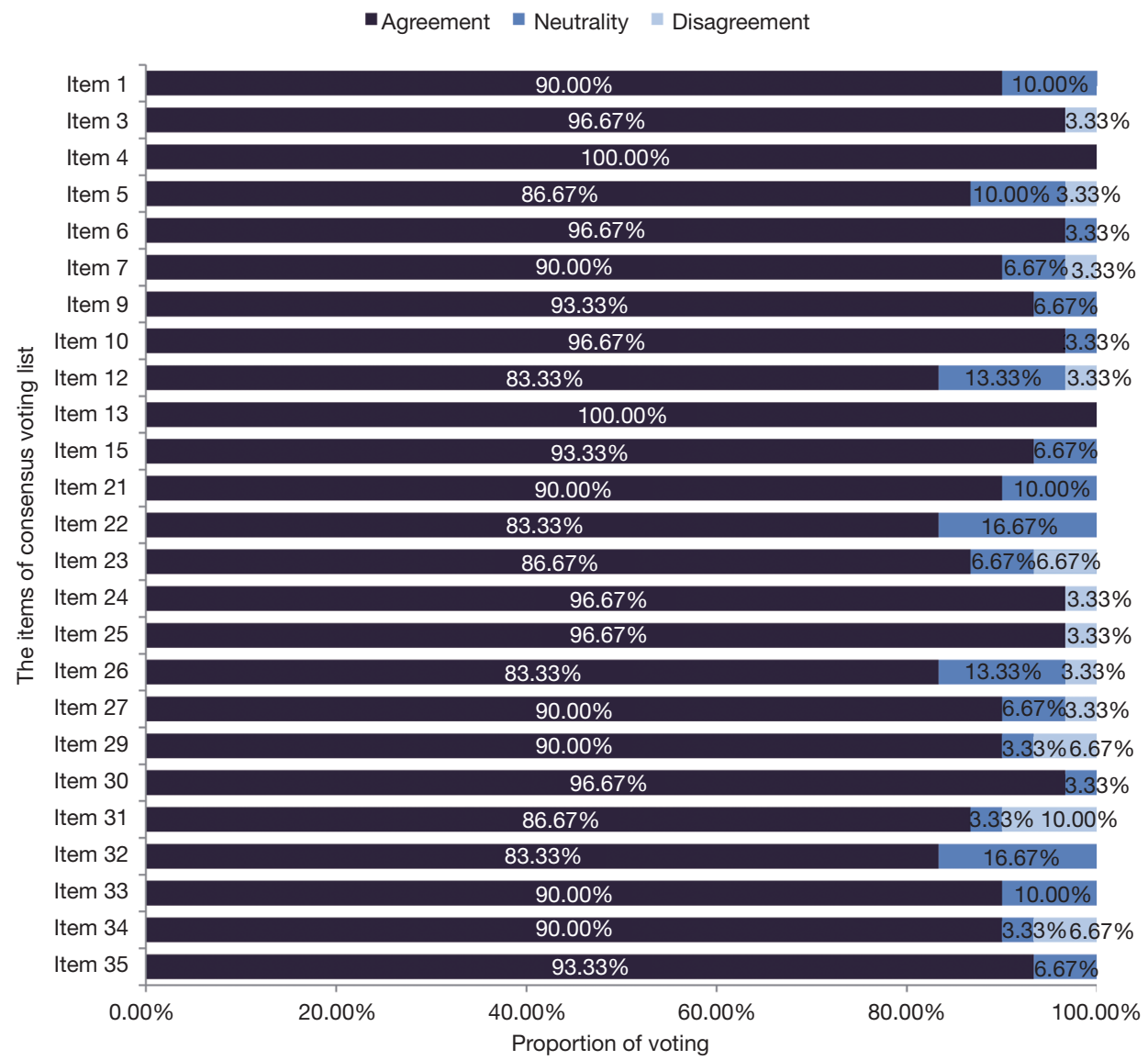

Figure 2 Results of the first round of Delphi survey.

whether acupuncture can influence the objective arrhythmia parameters. This may be the main reason for the hesitation of experts.

In view of the unique theory of TCM, experts emphasized that the principle of syndrome differentiation and treatment should be paid attention during the acupuncture treatment of arrhythmia, which is one of the basic principles of individualized treatment (36). On the other hand, the selection of acupoints is the crucial part of acupuncture treatment, especially the specific acupoints, which may directly affect the effectiveness (37-39). Based on the theory of acupuncture, specific acupoints are summarized with the specified label in the fourteen meridians according to their special therapeutic effects. Therefore, the assistant team specifically designed four items to discuss this issue. Experts highlight the application of specific acupoints and agree that specific acupoints should be given more priority during acupuncture treatment of arrhythmia. The recommended specific points include Neiguan, Xinshu, Danzhong and
Shenmen in this consensus. Consistent with the results of our consensus, a meta-analysis mentioned that 10 out of 13 studies mainly selected Neiguan, Xinshu and Shenmen for arrhythmia (12). In the management of CA, Neiguan is a commonly used specific acupoint. It has been demonstrated that electro-acupuncture on Neiguan point can lessen the myocardial injury by influencing $\mathrm{Ca}^{2+}$ key regulators (40), and protect the heart by regulating amino acid and energy metabolisms (41).

In addition to the acupoint selection, other acupuncture parameters (such as the number of acupoints, the frequency of acupuncture and the duration of needle retention) are of great concern to acupuncturists and can also affect the effectiveness. However, the appropriate parameters for these factors are often ignored and difficult to be verified in the randomized controlled trial. Delphi survey has a superiority that it can even be carried out securely while current evidence is insufficient in a certain field (42-44). We achieved consensuses on these parameters via Delphi survey. 


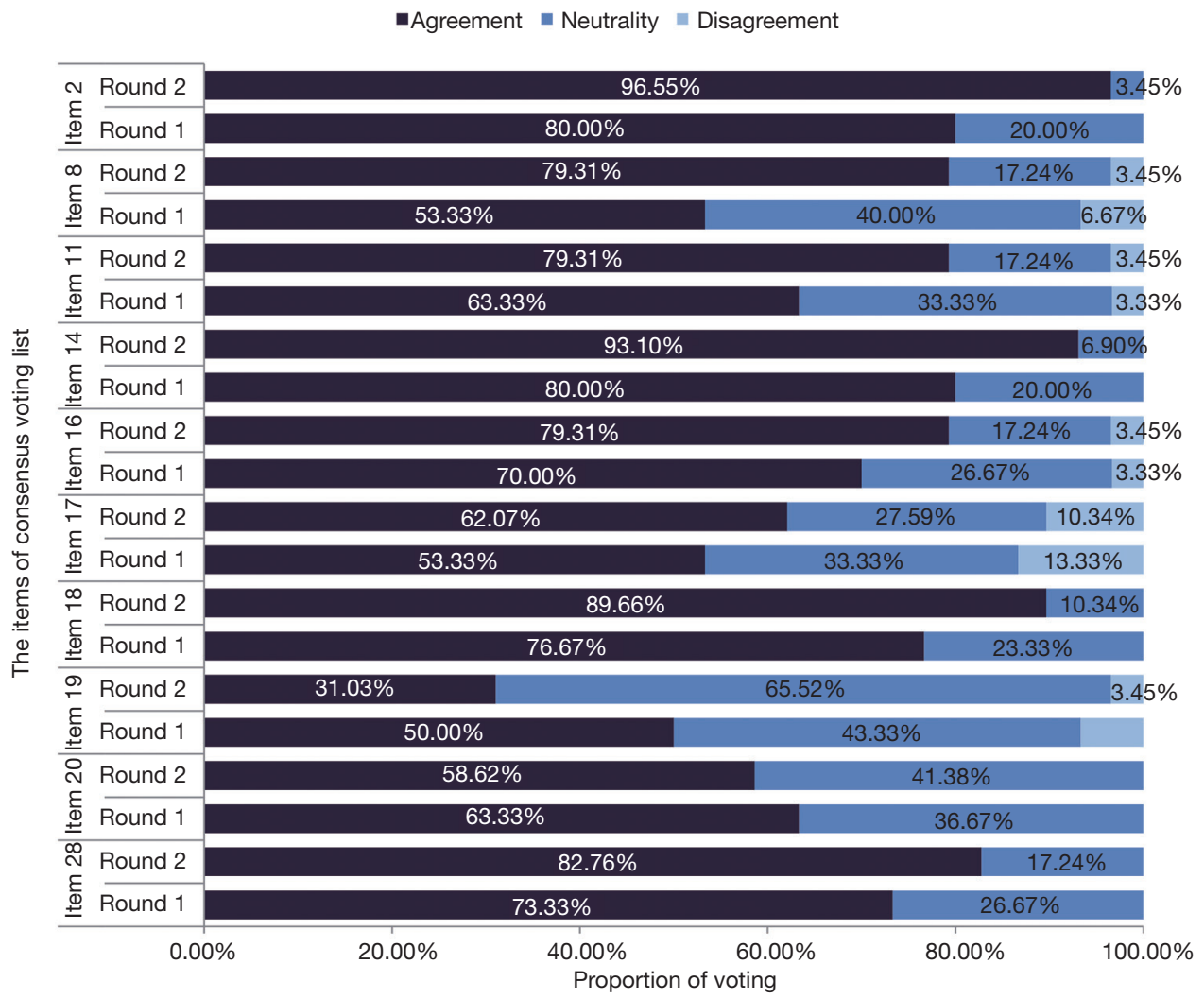

Figure 3 Results of the second round of Delphi survey.

More than $80 \%$ of the experts deem that it is reasonable to select 3-5 acupoints, retain 30 minutes, and 3 times a week. Since the Delphi survey can scientifically synthesize experts' opinions and deal with controversial clinical issues (45), our consensuses on these parameters are pragmatic for acupuncturists.

Arrhythmia is a kind of chronic disease that needs longterm management. In view of this, there is no wonder that safety should be one of the major concerns of patients and clinicians. Patients with arrhythmia are plagued by the increased risks of adverse effects from antiarrhythmic drugs, especially after long-term utilization. It should be noticed that one of the superiorities of acupuncture is safety. Ninety percent of experts agree that acupuncture has fewer adverse effects on the treatment of arrhythmia, which was similar to the previous studies $(12,26,35)$. Therefore, acupuncture may be a safer option for patients with arrhythmia.

There were many controversies relating to the effectiveness of acupuncture in the treatment of patients with atrial fibrillation or underwent invasive interventions. One systematic review demonstrated that acupuncture could bring benefits to patients with atrial fibrillation, such as increasing the rate of atrial fibrillation cardioversion to normal sinus rhythm (NSR), decreasing the time of atrial fibrillation cardioversion to NSR, reducing the heart rate (26). However, some experts retain a neutral or disagreed perspective on this aspect owing to the poor quality of studies and lack of clinical practice experience. Besides, fewer experts agree that acupuncture can reduce the recurrence rate or the incidence of relevant cardiovascular events after the invasive therapies. A major reason is the lack of available evidence on the effectiveness of acupuncture. Moreover, several experts honestly responded that they had limited experience of acupuncture in treating patients who underwent the invasive therapies. Since these patients generally had more serious conditions, cardiologists are their first choice, but not acupuncturists. Nevertheless, multidisciplinary treatment may provide more potentials for the management of arrhythmia, according to the remarkable efficacy of acupuncture in arrhythmia and the high agreement among panelists. Therefore, more highquality clinical studies are urgently required to fill this gap 


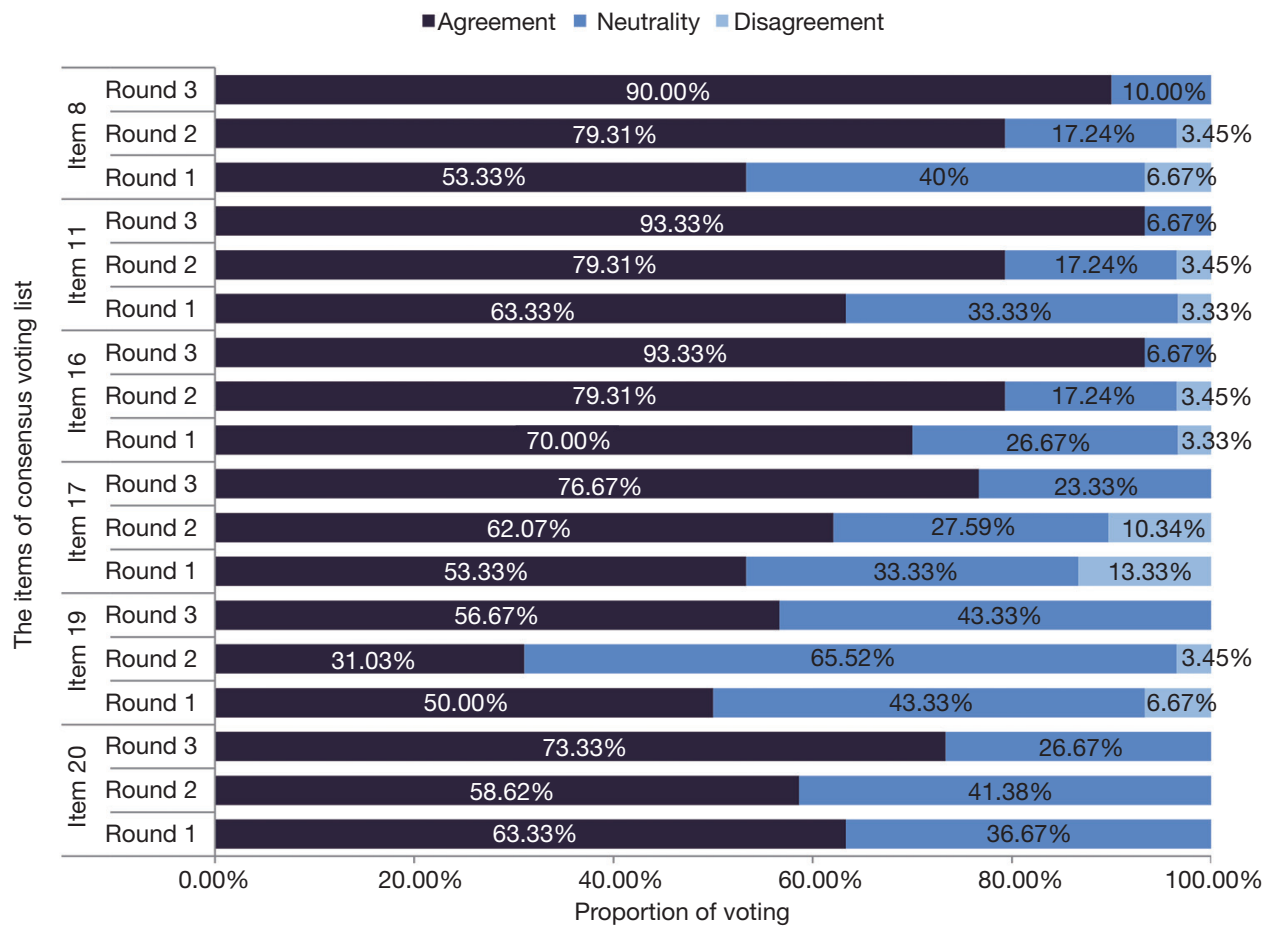

Figure 4 Results of the third round of Delphi survey.

and further verify the effectiveness of acupuncture.

This consensus provides a comprehensive comprehending for acupuncture in the treatment of arrhythmia by the integration of classical theories of TCM, clinical experience and literature review. Acupuncture is more inclined to empirical medicine, and the experience accumulated by acupuncturists plays an important role in the development of acupuncture. One of the highlights of this study is that the two-round clinical issue investigation was conducted prior to Delphi survey. The clinical issues, which attract the most attention, were specially collected from clinical specialists. During the clinical issue investigation, the feedbacks from clinical specialists were fully utilized in the development of the expert consensus voting list for Delphi survey. Furthermore, during the Delphi survey, another panel of experts were invited, who were more authoritative and experienced than the members in the clinical investigation stage. These experts were responsible for resolving controversial issues collected in the clinical investigation to provide guidance for clinical practice. These highlights will contribute to improve the practicality and accuracy of our consensus.

There are some limitations in this study. Despite both panels are carefully selected, these experts are all from
China, which limits the consensus extrapolation to other countries. Second, the available evidence cannot provide strong support for the consensus items due to the lowquality literature, which also promotes the development of our consensus. Third, it puts forward relevant suggestions on acupuncture strategies from a macroscopic perspective, and the therapeutic strategies for different subtypes of arrhythmias need to be further studied. Fourth, only veteran acupuncture experts were invited during Delphi survey, since this consensus aimed to formulate an optimal acupuncture strategy. However, experts in the field of arrhythmia should be invited in the future to improve the accuracy of the consensus.

\section{Conclusions}

In the case of insufficient evidence on clinical studies, the consensus generates an optimized acupuncture strategy, which provides a possibility to improve the management of arrhythmias. Our consensus propounds pragmatic recommendations and may be a useful tool for clinician decisions on the management of arrhythmia. Overall, acupuncture is one of the potential adjunctive therapies in arrhythmia and worth striving for. 


\section{Acknowledgments}

We wish to thank all members of the clinical panel and the consensus panel for carefully completing relevant questionnaires and sharing constructive suggestions. We would like to thank Prof. Fisher for his help in polishing our paper.

Funding: This work was supported by the National Key Research and Development Program of China (2019YFC1712102).

\section{Footnote}

Data Sharing Statement: Available at https://dx.doi. org/10.21037/cdt-21-201

Peer Review File: Available at https://dx.doi.org/10.21037/ cdt-21-201

Conflicts of Interest: All authors have completed the ICMJE uniform disclosure form (available at https://dx.doi. org/10.21037/cdt-21-201). The authors have no conflicts of interest to declare.

Ethical Statement: The authors are accountable for all aspects of the work in ensuring that questions related to the accuracy or integrity of any part of the work are appropriately investigated and resolved.

Open Access Statement: This is an Open Access article distributed in accordance with the Creative Commons Attribution-NonCommercial-NoDerivs 4.0 International License (CC BY-NC-ND 4.0), which permits the noncommercial replication and distribution of the article with the strict proviso that no changes or edits are made and the original work is properly cited (including links to both the formal publication through the relevant DOI and the license). See: https://creativecommons.org/licenses/by-nc-nd/4.0/.

\section{References}

1. Zheng J, Zhang J, Danioko S, et al. A 12-lead electrocardiogram database for arrhythmia research covering more than 10,000 patients. Sci Data 2020;7:48.

2. Bogle BM, Ning H, Mehrotra S, et al. Lifetime Risk for Sudden Cardiac Death in the Community. J Am Heart Assoc 2016;5:002398.

3. Arrhythmia treatment in the 21st Century? Eur Heart J
2019;40:797-8.

4. Lau DH, Nattel S, Kalman JM, et al. Modifiable Risk Factors and Atrial Fibrillation. Circulation 2017;136:583-96.

5. Laforest B, Dai W, Tyan L, et al. Atrial fibrillation risk loci interact to modulate $\mathrm{Ca} 2+-$ dependent atrial rhythm homeostasis. J Clin Invest 2019;129:4937-50.

6. Nielsen JB, Fritsche LG, Zhou W, et al. Genome-wide Study of Atrial Fibrillation Identifies Seven Risk Loci and Highlights Biological Pathways and Regulatory Elements Involved in Cardiac Development. Am J Hum Genet 2018;102:103-15.

7. D'Alessandro A, Boeckelmann I, Hammwhöner M, et al. Nicotine, cigarette smoking and cardiac arrhythmia: an overview. Eur J Prev Cardiol 2012;19:297-305.

8. Pathak RK, Mahajan R, Lau DH, et al. Sleep Apnea and Cardiac Arrhythmia: A Timely Wake-Up Call! Sleep 2015;38:1005-6.

9. Cai P, Zhong W, Wang Y, et al. Effects of whitecoat, masked and sustained hypertension on coronary artery stenosis and cardiac arrhythmia. Hypertens Res 2020;43:121-31.

10. Lavie CJ, Pandey A, Lau DH, et al. Obesity and Atrial Fibrillation Prevalence, Pathogenesis, and Prognosis: Effects of Weight Loss and Exercise. J Am Coll Cardiol 2017;70:2022-35.

11. Donahue JK. Current state of the art for cardiac arrhythmia gene therapy. Pharmacol Ther 2017;176:60-5.

12. Li Y, Barajas-Martinez H, Li B, et al. Comparative Effectiveness of Acupuncture and Antiarrhythmic Drugs for the Prevention of Cardiac Arrhythmias: A Systematic Review and Meta-analysis of Randomized Controlled Trials. Front Physiol 2017;8:358.

13. Nattel S, Andrade J, Macle L, et al. New directions in cardiac arrhythmia management: present challenges and future solutions. Can J Cardiol 2014;30:S420-30.

14. Andrade JG, Rivard L, Macle L. The past, the present, and the future of cardiac arrhythmia ablation. Can J Cardiol 2014;30:S431-41.

15. Lomuscio A, Belletti S, Battezzati PM, et al. Efficacy of acupuncture in preventing atrial fibrillation recurrences after electrical cardioversion. J Cardiovasc Electrophysiol 2011;22:241-7.

16. Holmqvist F, Kesek M, Englund A, et al. A decade of catheter ablation of cardiac arrhythmias in Sweden: ablation practices and outcomes. Eur Heart J 2019;40:820-30.

17. Cosedis Nielsen J, Johannessen A, Raatikainen P, et al. 
Radiofrequency ablation as initial therapy in paroxysmal atrial fibrillation. N Engl J Med 2012;367:1587-95.

18. Mont L, Bisbal F, Hernández-Madrid A, et al. Catheter ablation vs. antiarrhythmic drug treatment of persistent atrial fibrillation: a multicentre, randomized, controlled trial (SARA study). Eur Heart J 2014;35:501-7.

19. Dorman HG, van Opstal JM, Stevenhagen J, et al. Conductor externalization of the Riata internal cardioverter defibrillator lead: tip of the iceberg? Report of three cases and review of literature. Europace 2012;14:1161-4.

20. Kääb S. Genetics of sudden cardiac death - An epidemiologic perspective. Int J Cardiol 2017;237:42-4.

21. Takamiya T, Kubo Y, Benharash P, et al. Effect of electroacupuncture on porcine cardiac excitability induced by left stellate ganglion stimulation. Auton Neurosci 2018;213:15-22

22. Cao X, Lu S, Ohara H, et al. Beneficial and Adverse Effects of Electro-acupuncture Assessed in the Canine Chronic Atrio-ventricular Block Model Having Severe Hypertension and Chronic Heart Failure. Acupunct Electrother Res 2015;40:87-99.

23. Gao J, Zhao Y, Wang Y, et al. Anti-arrhythmic effect of acupuncture pretreatment in the rats subjected to simulative global ischemia and reperfusion--involvement of intracellular $\mathrm{Ca} 2+$ and connexin 43. BMC Complement Altern Med 2015;15:5.

24. Yin J, Yang M, Yu S, et al. Effect of acupuncture at Neiguan point combined with amiodarone therapy on early recurrence after pulmonary vein electrical isolation in patients with persistent atrial fibrillation. J Cardiovasc Electrophysiol 2019;30:910-7.

25. Wang G, Tian Y, Jia S, et al. Acupuncture regulates the heart rate variability. J Acupunct Meridian Stud 2015;8:94-8.

26. Fei Y, Fei R, Zhang J, et al. Systematic Evaluation of Efficacy and Safety of Acupuncture Treatment for Patients with Atrial Fibrillation. Open Access Maced J Med Sci 2019;7:461-6.

27. Kim TH, Choi TY, Lee MS, et al. Acupuncture treatment for cardiac arrhythmias: a systematic review of randomized controlled trials. Int J Cardiol 2011;149:263-5.

28. Shi GX, Yang XM, Liu CZ, et al. Factors contributing to therapeutic effects evaluated in acupuncture clinical trials. Trials 2012;13:42.

29. Hohmann E, Cote MP, Brand JC. Research Pearls: Expert Consensus Based Evidence Using the Delphi Method. Arthroscopy 2018;34:3278-82.

30. McMillan SS, King M, Tully MP. How to use the nominal group and Delphi techniques. Int J Clin Pharm 2016;38:655-62.

31. Sun N, Wang LQ, Shao JK, et al. An expert consensus to standardize acupuncture treatment for knee osteoarthritis. Acupunct Med 2020;38:327-34.

32. Hohmann E, Angelo R, Arciero R, et al. Degenerative Meniscus Lesions: An Expert Consensus Statement Using the Modified Delphi Technique. Arthroscopy 2020;36:501-12.

33. Vonk Noordegraaf A, Huirne JA, Brölmann HA, et al. Multidisciplinary convalescence recommendations after gynaecological surgery: a modified Delphi method among experts. BJOG 2011;118:1557-67.

34. Ravelli A, Consolaro A, Horneff G, et al. Treating juvenile idiopathic arthritis to target: recommendations of an international task force. Ann Rheum Dis 2018;77:819-28.

35. Liu J, Li SN, Liu L, et al. Conventional Acupuncture for Cardiac Arrhythmia: A Systematic Review of Randomized Controlled Trials. Chin J Integr Med 2018;24:218-26.

36. Cao H, Bourchier S, Liu J. Does Syndrome Differentiation Matter? A Meta-Analysis of Randomized Controlled Trials in Cochrane Reviews of Acupuncture. Med Acupunct 2012;24:68-76.

37. Xing JJ, Zeng BY, Li J, et al. Acupuncture point specificity. Int Rev Neurobiol 2013;111:49-65.

38. Zhao L, Chen J, Liu CZ, et al. A review of acupoint specificity research in china: status quo and prospects. Evid Based Complement Alternat Med 2012;2012:543943.

39. Hwang YC, Lee YS, Ryu Y, et al. Statistical inference of acupoint specificity: forward and reverse inference. Integr Med Res 2020;9:17-20.

40. Dai QF, Gao JH, Xin JJ, et al. The Role of Adenosine A2b Receptor in Mediating the Cardioprotection of Electroacupuncture Pretreatment via Influencing Ca2+ Key Regulators. Evid Based Complement Alternat Med 2019;2019:6721286.

41. Zhang HR, Tao JL, Bai H, et al. Changes in the Serum Metabolome of Acute Myocardial Ischemia Rat Pretreatment with Electroacupuncture. Am J Chin Med 2019;47:1025-41.

42. Keeney S, Hasson F, McKenna H. Consulting the oracle: ten lessons from using the Delphi technique in nursing research. J Adv Nurs 2006;53:205-12.

43. Sandrey MA, Bulger SM. The Delphi Method: An Approach for Facilitating Evidence Based Practice in Athletic Training. Athletic Training Education Journal 
2008;3:135-42.

44. Powell C. The Delphi technique: myths and realities. J Adv Nurs 2003;41:376-82.

Cite this article as: Li JL, Wang LQ, Zhang N, Su XT, Lin Y, Yang JW, Shi GX, Liu CZ. Acupuncture as an adjunctive therapy for arrhythmia: a Delphi expert consensus survey. Cardiovasc Diagn Ther 2021;11(5):1067-1079. doi: 10.21037/ cdt-21-201
45. Hsu CC, Sandford BA. The delphi technique: Making sense of consensus. Practical Assessment, Research, and Evaluation 2007;12:article 10. 